\title{
Traduire
}

Revue française de la traduction

$214 \mid 2007$

L'art de l'invisible

\section{Le projet lexALP : quand terminologues, juristes et politiques travaillent en quatre langues sur l'aménagement du territoire alpin}

\section{Élisabeth Lavault-Olléon et Claire Allignol}

\section{(2) OpenEdition \\ Journals}

Édition électronique

URL : http://journals.openedition.org/traduire/1399

DOI : $10.4000 /$ traduire.1399

ISSN : 2272-9992

Éditeur

Société française des traducteurs

\section{Édition imprimée}

Date de publication : 1 juin 2007

Pagination : 29-49

ISSN : 0395-773X

\section{Référence électronique}

Élisabeth Lavault-Olléon et Claire Allignol, « Le projet lexALP : quand terminologues, juristes et politiques travaillent en quatre langues sur l'aménagement du territoire alpin », Traduire [En ligne], 214 | 2007, mis en ligne le 01 juin 2007, consulté le 01 mai 2019. URL : http://

journals.openedition.org/traduire/1399; DOI : 10.4000/traduire.1399 


\title{
Le projet lexALP : quand terminologues, juristes et politiques travaillent en quatre langues sur l'aménagement du territoire alpin
}

\author{
Élisabeth Lavault-Olléon et Claire Allignol
}

Université Stendhal, Grenoble, Groupe de recherche multilingue en traduction spécialisée (GREMUTS)

Les traducteurs d'aujourd'hui ne peuvent plus travailler sans ressources en ligne. Les grandes bases de données terminologiques comme IATE, le Grand dictionnaire terminologique ou Termium, auxquelles s'ajoutent d'innombrables glossaires et dictionnaires spécialisés accessibles sur la Toile, sont devenues indispensables. Une nouvelle ressource est présentée dans cet article, la plate-forme LexALP. Son originalité : une thématique spécifique (l'aménagement du territoire), un territoire (l'arc alpin), et un traitement des concepts à différents niveaux juridiques grâce à un système informatique novateur.

\section{Le projet LexALP}

Le projet LexALP est mis en œuvre depuis 2005 par une équipe pluridisciplinaire internationale des pays de l'arc alpin. Il est financé en partie par l'Union européenne (programme INTERREG IIIB « Espace alpin »), et soutenu par des organismes publics locaux (régions alpines) et nationaux (ministères concernés). À l'origine du projet : l'existence d'un accord cadre supranational transalpin, la Convention alpine, dont la terminologie nécessite une harmonisation. 
L'objectif du projet est double : préciser et harmoniser la terminologie juridique utilisée par la Convention alpine en allemand, français, italien et slovène dans les domaines clés de l'aménagement du territoire et du développement durable ; parallèlement, proposer un ensemble de ressources linguistiques en ligne qui assureront la cohérence de la terminologie quadrilingue employée dans les instances supranationales des Alpes. Les traducteurs travaillant sur ces thématiques dans l'espace alpin seront les premiers bénéficiaires de la plateforme LexALP qui sera également utile aux juristes et personnels administratifs des collectivités territoriales alpines et rhône-alpines.

La Convention alpine, les partenaires du projet et la méthodologie employée en terminologie seront ici présentés successivement. Quelques exemples illustreront à la fois la difficulté des travaux terminologiques en cours et l'intérêt qu'ils peuvent présenter pour les traducteurs.

\section{La Convention alpine}

La Convention alpine est un accord international " visant la sauvegarde de l'écosystème naturel ainsi que la promotion du développement durable des Alpes, en protégeant les intérêts économiques et culturels des populations qui y habitent et des pays adhérents ${ }^{(1)}$ ». La décision d'élaborer un tel texte a été prise en 1989 lors d'une réunion des ministres de l'Environnement de sept pays et de représentants de la Communauté européenne. À cette époque, les futurs signataires avaient pris pleinement conscience des menaces pesant sur l'espace alpin et ils étaient déterminés à allier leurs forces pour protéger et développer cette zone qui représente, au cœur de l'Europe, l'un des plus grands espaces naturels d'un seul tenant ainsi qu'un espace culturel, économique et récréatif unique, traversé par des axes de communication essentiels à la vie économique européenne. La Convention a été signée en France en 1991, par l'Allemagne, l'Autriche, la France, l'Italie, le Liechtenstein, la Suisse, la Slovénie et

(1) http://www.convenzionedellealpi.org/page1_fr.htm 
la Communauté économique européenne ${ }^{(2)}$. La Convention alpine a pour but de promouvoir le développement économique durable de l'arc alpin, zone délimitée à la fois sur des critères géographiques et administratifs, et de favoriser des mesures de développement adaptées aux spécificités des zones de montagne. Cette Convention comporte neuf protocoles qui traitent plus spécifiquement de l'aménagement du territoire et du développement durable, des transports, du tourisme, de l'agriculture de montagne, de la protection des sols, de la protection de la nature et de l'environnement, de l'énergie, et enfin des forêts de montagne, un dernier protocole étant consacré au règlement des différends. Ces protocoles sont venus s'ajouter peu à peu à la Convention alpine. À titre d'exemple, si la Convention a été signée en 1991, le protocole d'application sur la protection des sols date de 1998.

Si l'ensemble de l'espace alpin présente des caractéristiques morphologiques communes (c'est une zone montagneuse), sa gestion souffre d'une grande hétérogénéité politique, administrative et juridique. La diversité et la multiplicité des réglementations applicables à cette zone ont rendu nécessaire l'élaboration de cet accord cadre pour les Alpes.

Ce texte accorde une grande importance à la préservation de l'environnement et au développement durable de l'arc alpin, sans oublier les activités humaines. La Convention alpine ne vise pas à faire des Alpes une grande réserve naturelle, mais à promouvoir toute la zone, en favorisant aussi bien des activités traditionnelles comme l'agriculture de montagne ou l'exploitation raisonnée des forêts que des activités plus récentes comme le tourisme, la production d'énergie, la mise en place de transports propres et les technologies avancées... Le développement durable et dynamique ainsi prôné nécessite une coopération et des activités de recherche scientifique transfrontalières.

Les États signataires ont ratifié la Convention, qui entre ainsi petit à petit en application dans les différents pays. Tous les textes existent en

(2) Monaco a rejoint la Convention en 1999. 
quatre langues, qui sont le français, l'allemand, l'italien et le slovène. L'anglais n'est pas une langue de la Convention alpine, puisqu'aucun pays alpin n'est anglophone. La langue de rédaction de chaque texte est celle du pays dans lequel le texte en question a été signé. Par exemple, le protocole d'application de la Convention alpine concernant l'agriculture de montagne a été signé à Chambéry en France. La langue de rédaction était donc le français, les trois autres versions étant des traductions.

Le fait que trois des textes soient toujours des traductions est à l'origine de certaines anomalies de formulation. Par exemple, " espace de protection de la nature " est une expression possible en français, mais qui n'existe dans aucun texte législatif national ou européen. Il y a ainsi des anomalies terminologiques qui ne sont pas sans portée juridique et qu'il convient de mettre en évidence. Les organisations participant au développement de la région alpine, comme le Secrétariat permanent de la Convention alpine, souhaitent que les termes juridiques utilisés soient fiables et clairement définis. C'est le but de l'harmonisation terminologique effectuée grâce au projet LexALP.

\section{Genèse et objectifs du projet LexALP}

C'est en Italie que s'est manifesté le besoin d'une base de données terminologique pour la Convention alpine. En effet, l'organisation décentralisée de l'Italie, avec des régions dotées d'une large autonomie, dont certaines sont bilingues comme la province autonome de Bolzano, place les juristes devant certains problèmes liés à la terminologie. En matière de législation, on distingue en Italie les compétences exclusives de l'État, les compétences exclusives des régions, les compétences partagées entre l'État et les régions. Les régions italiennes, qui n'ont pas toutes le même statut, ont une compétence législative pour les affaires strictement régionales et les régions qui se trouvent sur le territoire alpin sont directement concernées par la Convention alpine. D'une région à l'autre, on peut observer des usages linguistiques différents, un terme juridique ayant une signification spécifique dans une 
région pouvant en avoir une autre dans la région voisine. Les incertitudes terminologiques sont gênantes lorsque deux régions italiennes voisines prennent des mesures, chacune à son niveau, pour mettre en pratique la Convention alpine. Par exemple, un texte administratif du Val d'Aoste peut utiliser un terme juridique dans un autre sens qu'une loi régionale du Trentin-Haut-Adige.

Les juristes italiens de ces régions sont confrontés à ces différences d'emploi des termes et sont sensibles à l'insécurité juridique qui peut en résulter. C'est la raison pour laquelle ils ont demandé la création d'une telle base de données terminologique, destinée d'une part aux traducteurs et responsables administratifs et politiques travaillant sur des sujets couverts par la Convention alpine, amenés à rédiger des textes administratifs et réglementaires, d'autre part aux juristes, italiens principalement, qui rédigent des textes législatifs au niveau régional.

Grâce à cette base de données, les imprécisions et incertitudes juridiques devraient progressivement disparaitre. La demande italienne a conduit à une prise de conscience de différences sémantiques potentielles entre des termes employés dans des pays de langues communes (allemand en Suisse, Allemagne, Autriche et Liechtenstein ; français en Suisse, France et à Monaco) mais de systèmes juridiques différents. L'Union européenne, signataire de la Convention et de ses protocoles, a décidé de soutenir la constitution de cette base de données.

\section{Gestion du projet et partenaires}

Le projet est piloté depuis l'Italie par l'Académie européenne de Bolzano. Il s'agit d'une structure de droit privé, un institut de recherche qui réalise de nombreuses tâches pour le gouvernement régional, organise des séminaires de formation et publie des manuels et des dictionnaires. Un groupe de six personnes, dont une spécialiste d'informatique et de traitement des langues naturelles, y travaille pour le projet. 
Les autres partenaires italiens sont le ministère des Affaires régionales et le ministère de l'Environnement et de l'Aménagement du territoire et de la Mer à Rome, ainsi que le Service des relations européennes de la Région autonome Frioul-Vénétie-Julienne à Trieste.

En Allemagne, c'est l'institut de traduction SDI (Sprachen- und Dolmetscherinstitut) à Munich qui s'est engagé dans le projet, en y associant ses enseignants et ses étudiants.

En France, l'université de Grenoble apporte son concours dans deux domaines distincts. Les spécialistes de terminologie et de traduction de l'université Stendhal (notamment le Groupe de recherche multilingue en traduction spécialisée, GREMUTS ${ }^{(3)}$, associé au LIDILEM, Laboratoire de linguistique et didactique des langues étrangères et maternelles) s'occupent du travail terminologique proprement dit. C'est l'université Joseph Fourier, par le biais de son laboratoire Communication langagière et interaction personne-système (CLIPSIMAG) qui est chargée du volet informatique pour l'ensemble des ressources, et notamment pour la base de données, fondée sur un système novateur appelé Papillon.

La Suisse figure également parmi les partenaires du projet à travers la section de terminologie de la Chancellerie de la Confédération suisse à Berne.

Sur huit partenaires, quatre sont italiens, ce qui reflète bien l'importance que revêt aux yeux de l'Italie la constitution de cette base de données terminologiques. On pourrait s'étonner de ne pas trouver de partenaire slovène dans la liste. En réalité, la Slovénie est représentée à Bolzano par une terminologue qui travaille à plein temps pour le projet LexALP, efficacement épaulée par des juristes slovènes.

Le projet LexALP bénéficie également des conseils d'un certain nombre d'observateurs : le Secrétariat permanent de la Convention 
alpine (Innsbruck, Autriche), la direction de l'Environnement de la Région autonome de la Vallée d'Aoste (Italie), le bureau des questions linguistiques de la Province autonome de Bolzano/Bozen (Italie), le ministère fédéral de l'Agriculture, des Forêts, de l'Environnement et de l'Eau (Vienne, Autriche) et enfin la Délégation générale à la langue française et aux langues de France (DGLFLF, ministère de la Culture et de la Communication, Paris), qui nous apporte une aide précieuse en consultant au besoin les commissions ministérielles de néologie et de terminologie sur les termes délicats.

L'Autriche, qui accueille le Secrétariat permanent de la Convention alpine, ne participe pas directement aux travaux terminologiques, mais le Secrétariat permanent est représenté aux réunions de travail et contribue de manière constructive aux travaux d'harmonisation.

Chaque partenaire a la charge d'un ensemble de termes, choisis par domaine. Le travail de notre équipe consiste à établir des fiches terminologiques pour chaque terme, à chaque niveau juridique et en trois langues (français, allemand et italien), les législations de la Suisse et de l'Autriche n'étant pas de notre compétence, ni le slovène, traité à Bolzano. Loriginalité de notre participation tient à son intégration dans la formation universitaire des traducteurs : en effet, les étudiants de dernière année du Master "Traduction spécialisée multilingue " travaillent en binômes sur des séries de termes en trois langues (français, italien, allemand), encadrés par une doctorante chargée de recherche sur le projet.

\section{Difficultés et contraintes}

Un projet tel que LexALP, qui fait intervenir des partenaires différents dans plusieurs pays, et qui marie intérêts scientifiques et contraintes politiques, n'est pas développé uniquement selon les critères des linguistes. Les politiques et les bailleurs de fonds ont leur mot à dire et jouent un rôle déterminant. Ils n'ont pas toujours les mêmes vues que les linguistes. Durant la phase initiale du projet qui a défini la 
méthodologie, certains semblaient trouver que les discussions sur la méthodologie terminologique, notamment sur la sélection d'un gros corpus, ainsi que le travail d'alignement et d'extraction ralentissaient le projet. Par ailleurs, les juristes ont besoin de temps pour étudier les glossaires terminés en vue de l'harmonisation et ils manifestent une certaine impatience devant la lenteur des travaux des terminologues, eux-mêmes empêtrés dans leurs niveaux juridiques superposés et confrontés à un serveur informatique parfois surchargé.

Du point de vue de l'organisation, la masse de contraintes administratives et de contrôles financiers rend la gestion de projet fastidieuse. Chaque partenaire doit avancer les fonds nécessaires et faire certifier les dépenses non seulement par son propre service comptable, mais aussi par un organisme de certification indépendant, dont le feu vert est indispensable pour obtenir les remboursements dans les délais, ce qui est source de préoccupation et de tension pour le chef de projet. Mais il est vrai que des procédures rigoureuses sont indispensables pour garantir l'utilisation satisfaisante des fonds européens et que seule une grande rigueur permet de mener à bien de tels projets qui nourrissent les échanges et la coopération à l'intérieur de l'Europe.

Enfin, pour ce qui est des séances de travail, des problèmes d'intercompréhension se sont parfois fait sentir. Il a été décidé au départ que la langue de travail était l'anglais, connu de la majorité des participants, ce qui, pour un projet de terminologie quadrilingue excluant l'anglais, n'est pas le moindre des paradoxes, et reflète ironiquement bien des situations propres à l'Union européenne... Cela pose néanmoins problème à ceux des partenaires qui ne le maîtrisent pas bien. Lors des réunions de travail, le niveau d'anglais assez simple permet de suivre l'essentiel des débats, mais les comptes rendus de réunions, les plannings de travail, les rapports et tous les courriers électroniques, y compris les plus techniques, sont rédigés en anglais (ou plutôt en globish). Au fil de l'avancée du projet, et contrairement à ce qui se passait au départ, on s'aperçoit que, de plus en plus, les réunions se tiennent dans un joyeux mélange de langues. Lidée est que chacun puisse s'exprimer dans sa langue ou dans une langue qu'il connaît, les autres 
répondant dans leur langue maternelle ou dans une autre langue, certains traduisant, ou pas, et le slovène étant généralement exclu car non partagé par les interlocuteurs (la terminologue slovène est quadrilingue). Cela donne une polyphonie italo-germano-française qui fonctionne plus ou moins bien selon la composition des groupes de travail, mais qui correspond bien mieux qu'un anglais maladroit et non authentique à la conception multilingue et multiculturelle de l'Europe à laquelle les linguistes sont généralement attachés.

\section{Système informatique}

Le système informatique devait répondre à une exigence principale, formulée par les juristes italiens : les définitions à inclure dans la base de données doivent tenir compte des différents niveaux juridiques (Convention alpine, traités internationaux, textes européens, législation nationale, voire régionale), et la base de données doit permettre de visualiser ces différents niveaux. Ainsi, un terme de la Convention alpine doit avoir une définition pour la Convention alpine, bien entendu, mais il peut aussi en avoir une aux trois autres niveaux. La signification du terme doit être étudiée dans les traités internationaux pertinents pour les domaines concernés afin de proposer une définition pour ce niveau-là, et de même pour le niveau européen et pour le niveau national. Le but est de bien cerner les différences de signification d'un seul et même terme, lorsqu'il y en a.

Le laboratoire CLIPS-IMAG de l'université Joseph Fourier à Grenoble, à l'origine du système informatique, a conçu un outil capable de tenir compte de ces différents niveaux. Les termes sont reliés entre eux, à chaque niveau, au moyen de ce qui est appelé une " axie ", et que l'on pourrait décrire comme un "nœud » synthétisant un ensemble de marques sémantiques propres à un concept. Ainsi, lorsqu'un terme a été traité pour chacun des niveaux dans plusieurs langues, une axie est créée pour les réunir, à la condition expresse qu'il y ait identité de signification à un niveau juridique défini. 
Une axie peut à son tour être réunie à une autre axie, ce qui permet de créer des familles de termes apparentés, dans les quatre langues. Concrètement, ce système permet au traducteur de trouver, à partir du terme en langue de départ, l'équivalent lexical en langue d'arrivée correspondant au niveau juridique dans lequel se place sa traduction, et de connaître la définition de ce terme dans ce même système juridique. Pour un responsable administratif, l'avantage de cette base de données est qu'elle lui permet de constater s'il y a ou non des différences de signification ou d'emploi d'un terme aux différents niveaux. En effet, un responsable politique français, par exemple, devant rédiger un texte réglementaire sur la protection de la flore dans une région alpine transfrontalière, a tout intérêt à connaître les spécificités terminologiques des pays concernés, et à ne pas considérer que la terminologie française a une portée universelle.

Sur l'écran présenté ci-dessous en figure 1 se trouve l'interface de recherche de la plate-forme LexALP avec l'affichage de l'axie correspondant à une recherche sur le terme captage (domaine de la protection de l'eau). En faisant défiler le curseur sur la liste, on obtient la

\section{Figure 1}

\begin{tabular}{|c|c|c|}
\hline Lex $4 L$ & BANQUE DE TERMES & \\
\hline Utilisateur: & Adval & $\begin{array}{l}\text { 10: fracacaptage_de__eau polable.6475103.e } \\
\text { Telim: captage d'eau potable }\end{array}$ \\
\hline Languve: hrancisis $v$ & & 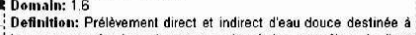 \\
\hline & $\sim$ contient & 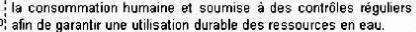 \\
\hline Consultation: & Toutes les langues $v$ & Coutext: Les Ettats membres recensent les eaux utilisées pour le \\
\hline $\begin{array}{l}\text { Ierme: } \\
\text { Scarce: }\end{array}$ & & 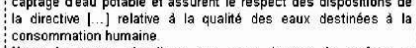 \\
\hline Gole: toutes les lang 2 & & $\begin{array}{l}\text { Note: Le terme s'applique aux eaux douces de surface en } \\
\text { souterraines. II existe des captages d'eaux differentes : potable. }\end{array}$ \\
\hline Go & Afficher $10 \sim$ resultats par page en urililsaut la fo & 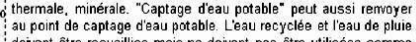 \\
\hline \begin{tabular}{|l} 
Recherche avancúe \\
Liste des detionnaires
\end{tabular} & 1 entryfies) retrieved. & $\begin{array}{l}\text { dowent être recuillies mais ne doivent pas âtre utilisées comme } \\
\text { sanitaire ou eau potable. }\end{array}$ \\
\hline 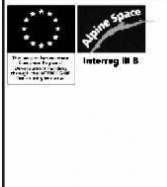 & 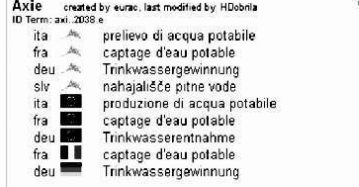 & \\
\hline & $\begin{array}{l}\text { Related to axile } \\
\text { slv } \\
\text { slv } \\
\text { vir pitne vode } \\
\text { vir pitne vode }\end{array}$ & \\
\hline
\end{tabular}


fiche correspondant à chaque terme, avec sa définition et son contexte. On peut également faire la recherche directement sur le terme en demandant les fiches dans les langues sélectionnées.

\section{Corpus}

Tout travail terminologique s'appuie sur un corpus : la base de celuici est la Convention alpine avec ses protocoles, dans les quatre langues alpines. La structure du corpus suit la thématique de ses neuf protocoles, depuis l'aménagement du territoire jusqu'aux transports, en passant par l'énergie, la protection des paysages, etc.

Le corpus LexALP comprend 3000 documents ( 20 millions de mots) sur quatre niveaux de législation :

- niveau de la Convention alpine,

- niveau du droit international,

- niveau du droit communautaire (européen),

- niveau national propre à chaque État, voire région (Allemagne, Autriche, France, Italie, Suisse, Slovénie, soit six niveaux).

Le corpus ne comprend pas de textes scientifiques (géographiques par exemple) mais seulement des textes juridiques ou réglementaires correspondant à chaque niveau à traiter, ceux-ci ayant été extraits de bases disponibles sur Internet pour les différentes législations. Tous ces documents réglementent les domaines de l'aménagement du territoire et du développement durable. Au total, ce sont bien neuf législations différentes qui sont abordées.

En droit international, il s'agit des principaux textes pertinents pour le projet : par exemple, la Convention de Rio (sur la diversité biologique, 1992), la Convention de Berne (sur la conservation de la vie sauvage en Europe, 1981), la Convention de Ramsar (sur les zones humides, 1971), le protocole de Londres (sur l'eau et la santé, 1999). Ces textes existent le plus souvent en plusieurs langues, ce qui permet de les aligner pour rechercher les mêmes articles dans les quatre langues. 
Le droit communautaire est composé non seulement des traités fondateurs mais surtout d'innombrables directives, règlements, décisions, recommandations, résolutions, etc. généralement disponibles dans les quatre langues.

Pour le droit national français, treize codes sur 60 ont été sélectionnés : construction et habitat, domaine de l'État, domaine public fluvial, douanes, environnement, forestier, général des collectivités locales, marchés publics, patrimoine, rural, tourisme, urbanisme, voirie routière. Cette sélection a été plus difficile à faire pour les pays à structure fédérale (Allemagne, Suisse, Autriche) qui ont différents niveaux juridiques sur leur territoire.

Le corpus a été étiqueté par domaines et inséré dans la plate-forme LexALP qui comprend un outil de recherche adapté pour les terminologues du projet. Pour les futurs utilisateurs, il sera possible d'afficher les extraits du corpus correspondant à la recherche en cours.

\section{Sélection des termes}

Notre proposition initiale était d'utiliser des logiciels d'extraction automatique de termes pour dégager une liste de candidats termes pertinents à partir de l'ensemble du corpus.

Finalement, les juristes italiens ont imposé leur point de vue : partir de la Convention alpine dans sa version italienne et extraire tous les termes et expressions sur les domaines concernés dans les quatre langues. Cela a permis de travailler à partir d'une liste assez resserrée de termes à traiter systématiquement aux différents niveaux, et à enrichir au fur et à mesure.

Linconvénient est que, du fait de la traduction, certains équivalents utilisés ne sont pas les termes reconnus. Il a donc fallu faire des recherches pour rétablir les bonnes équivalences, mais cela répondait exactement à l'objectif du projet : améliorer la terminologie utilisée par la Convention dans les quatre langues. L'harmonisation des termes a entraîné l'abandon de certains synonymes peu fréquents ou de for- 
mulations maladroites. Un autre inconvénient a été un découpage large, qui comprenait des unités phraséologiques dont la définition et le traitement aux quatre niveaux ne présentaient pas d'intérêt, mais qui pouvaient être utiles pour des traducteurs (exemple : "plan d'aménagement du paysage ", " aire de répartition d'une espèce migratrice ", "valorisation du patrimoine naturel "). Bien entendu, ces unités ne sont pas toujours découpées de la même façon selon les langues, ce qui a considérablement pimenté le travail.

La démarche imposée est finalement une démarche sémasiologique classique, à partir d'une liste. En travaillant sur le projet, nous constatons certains inconvénients liés à ce type de travail ponctuel et analytique terme à terme, qui nécessiterait une démarche critique audacieuse. Nous essayons de revenir aux concepts et de structurer chaque champ conceptuel en utilisant les possibilités données par les axies, mais le travail s'avère souvent plus lexicographique que terminographique : il nous est imposé de traiter des termes généraux qui ne connaissent pas de variations conceptuelles entre les niveaux juridiques mais mériteraient une étude lexicographique que nous n'avons pas le loisir de mener. Par exemple, dans le domaine de la protection de la nature, comment adopter une démarche terminologique sur des dénominations aussi vastes et proches que protection, sauvegarde, conservation ou aire, zone, site? En revanche les recherches montrent que zone humide, zone-tampon, forêt de montagne ou état de conservation favorable sont définis dans certaines législations et méritent un traitement terminologique précis.

\section{Structure des fiches}

Chaque fiche correspond à un niveau juridique (représenté par un drapeau), avec un contexte et une définition correspondant à ce niveau. Comme la Convention alpine contient peu de définitions, le travail consiste à trouver au niveau international ou communautaire une définition qui pourra être proposée dans les quatre langues de la Convention. Sur la fiche ci-dessous, qui traite le terme diversité biologique au 
niveau Convention alpine (AC), c'est la définition issue de la Convention de Rio qui a été retenue car c'est la définition internationale en vigueur. Le contexte vient, lui, de la Convention alpine. Sur la fiche traitant du même terme au niveau international ou européen, c'est un autre contexte qui s'affichera. Les sources des définitions et des contextes sont référencées selon une liste d'abréviations définie.

\section{Exemple : niveau Convention alpine}

diversité biologique, n.f.

LANGUAGE : fra, LEGAL SYSTEM: AC

ID Term : fra.diversité_biologique.1284144.e

Frequency : common

DOMAIN : 1.3 Espaces protégés

SYNONYM : biodiversité

DEFINITION : Variabilité des organismes vivants de toute origine y compris, entre autres, les écosystèmes terrestres, marins et autres écosystèmes aquatiques et les complexes écologiques dont ils font partie ; cela comprend la diversité au sein des espèces et entre espèces ainsi que celle des écosystèmes. [Document : CDB 1992, art. 2]

CONTEXT : Les politiques d'aménagement du territoire et de développement durable visent à harmoniser au moment opportun les intérêts économiques avec les exigences de protection de l'environnement, notamment en ce qui concerne [...] la sauvegarde et le rétablissement de l'équilibre écologique et de la diversité biologique des régions alpines [...]. [Document : Prot.ATDD., art.3, a]

NOTE : « diversité écologique », employé comme synonyme dans la Convention alpine, renvoie à un autre concept.

\section{Harmonisation}

Lorsque toutes les fiches correspondant à un thème sont terminées, le glossaire ainsi obtenu circule parmi les partenaires et c'est le groupe d'harmonisation qui est chargé de la validation définitive des propositions faites par les terminologues et les juristes du projet. 
Ce groupe comprend les représentants des partenaires du projet et les observateurs. Il s'appuie aussi sur les avis fournis par des spécialistes du développement durable et de l'aménagement du territoire externes au projet. À l'occasion des réunions officielles du Groupe d'harmonisation, ses membres évaluent, discutent et, si nécessaire, modifient les propositions d'harmonisation. Chaque terme est finalement validé officiellement en quatre langues et les résultats sont mis à la disposition d'un large public sur la plate-forme LexALP.

Voici un exemple montrant comment l'harmonisation peut fonctionner. Dans la Convention alpine, en plus du terme diversité biologique, on trouve aussi deux occurrences du terme diversité écologique. Lors de l'élaboration des fiches, ne trouvant pas d'autres occurrences de ce dernier dans la législation dont nous disposions, nous avons supposé que le terme diversité écologique était un synonyme de diversité biologique. Ce n'est qu'à la séance d'harmonisation où nous pensions éliminer ce terme au profit de diversité biologique qu'un expert a défendu la spécificité de ce terme qui désigne un concept différent : "diversité spécifique des écosystèmes au sein de l'ensemble plus large de la diversité biologique ». Le terme a donc été regroupé dans une axie à part, elle-même reliée à l'axie du concept "diversité biologique ".

\section{Études de cas}

Voici quelques exemples supplémentaires qui illustreront le type d'harmonisation qui a été effectué et les problèmes rencontrés lors du traitement terminologique.

Exemple 1

Area di protezione naturalistica/Riserva naturale

Naturschutzgebiet

Naravni rezervat

* Espace de protection de la nature

Ces quatre termes sont donnés comme équivalents dans la Convention alpine (AC). Cependant, aux niveaux international (INT), européen 
(EU) et national français (FR) ce sont les termes protection de la nature et espace protégé qui prédominent (et désignent des concepts différents, qui font déjà l'objet de fiches). En travaillant sur le contexte de ce terme dans la Convention alpine, y compris dans les autres langues, et en faisant des recherches, notamment dans le code de l'environnement, nous avons constaté que le terme désignant le concept en question était réserve naturelle, absent de la Convention. Après avoir obtenu l'avis ultime de l'expert terminologue du ministère de l'Écologie via la DGLFLF, c'est réserve naturelle qui a été proposé à la place de espace de protection de la nature pour les textes à venir.

Exemple 2

Area di protezione paesaggistica/Paesaggio protetto
Landschaftsschutzgebiet
Krajinski park
${ }^{*}$ Espace de protection du paysage

Ce terme français était lui aussi absent du corpus. C'est le terme paysage protégé qui a été retenu par le Groupe d'harmonisation.

Exemple 3

\section{Area protetta transfrontaliera \\ Grenzïberschreitendes Schutzgebiet}

Espace protégé transfrontalier

Ici, c'est la définition juridique du terme qui a été source de discussions animées car, pour certains terminologues italiens, par rapport à la Convention alpine (et en gardant à l'esprit le contexte des régions autonomes italiennes), le terme et son équivalent italien pouvaient désigner un "espace protégé par des pays ou régions (entités administratives infranationales) voisines ". Cette première définition, qui correspondait à un concept large, a été farouchement critiquée par les organismes nationaux, notamment la DGLFLF, pour lesquels transfrontalier ne peut s'utiliser qu'à propos d'une frontière entre deux États. La définition harmonisée est donc la suivante : « espace protégé par des pays de part et d'autre d'une frontière commune, permettant un continuum écologique et servant notamment de couloir pour de nombreuses espèces migrant à travers les frontières ». 
Exemple 4

\section{Paesaggio culturale \\ Kulturlandschaft \\ Kulturna krajina}

Paysage culturel

Au départ, ces termes étaient regroupés avec les termes paysage agraire, paysage cultivé et paysage rural. Dans ce groupe de trois, le dernier est hyperonyme des deux premiers car il fait référence à des activités agricoles mais intègre aussi la plurifonctionnalité des espaces concernés (tourisme, habitats d'urbains...), si bien que l'agriculture n'en est qu'une composante non exclusive. Dans un premier temps, nous avons rejeté le terme paysage culturel que nous ne pouvions qu'associer à la scène culturelle (dans le même type d'emploi que paysage audiovisuel). Le terme paysage cultural, bien référencé, semblait alors plus approprié. La contribution de la DGLFLF et de ses experts a permis d'isoler un autre concept, bien différent, celui de "paysage culturel " qui n'est pas forcément un paysage rural. En effet, depuis 1992, la Convention du patrimoine mondial de l'UNESCO est le premier instrument juridique international à reconnaître et à protéger les paysages culturels $s^{(4)}$, qui sont les " ouvrages combinés de la nature et de l'homme " désignés dans son article 1 .

(4) La formalisation et la formulation des critères que s'est donné l'UNESCO pour ces paysages culturels l'a conduite à en distinguer trois catégories majeures. 1. le paysage clairement défini, conçu et créé intentionnellement par l'homme, ce qui comprend les paysages de jardins et de parcs créés pour des raisons esthétiques qui sont souvent (mais pas toujours) associés à des constructions ou des ensembles religieux. 2. Le paysage essentiellement évolutif, qui résulte d'une exigence à l'origine sociale, économique, administrative et/ou religieuse et atteint sa forme actuelle par association avec et en réponse à son environnement naturel. Ce type de paysages reflète, dans leur forme et leur composition, ce processus évolutif, soit qu'il s'agisse d'un paysage relique (ou fossile), où cette évolution s'est arrêtée, soit qu'il s'agisse d'un paysage vivant qui conserve un rôle social actif dans la société contemporaine, étroitement associé au mode de vie traditionnel et dans lequel le processus évolutif continue. 3. La dernière catégorie est nommée paysage culturel " associatif " ou " en association ", parce que l'inclusion de ce type de paysage sur la Liste du patrimoine mondial se justifie par la force d'association des phénomènes religieux, artistiques ou culturels avec les éléments naturels, plutôt que par des traces culturelles tangibles qui peuvent être insignifiantes ou même inexistantes, (d'après E. Binet, ministère de l'Écologie). 


\section{Exemple 5}

Eau potable, acqua potabile, Trinkwasser : un cas facile ? Une définition lexicologique comme " eau qui peut être bue sans danger " n'est évidemment pas suffisante dans le cadre de la terminologie juridique. Il apparaît vite que le concept " eau potable " a subi une évolution au fil des législations européennes et internationales et que, de plus, les législations nationales ne découpent pas toutes le concept de la même façon. Ainsi dans le droit communautaire (dir. 98/83, art. 2), " eau potable " = " eaux destinées à la consommation humaine ", c'est-à-dire " a) toutes les eaux, soit en l'état, soit après traitement, destinées à la boisson, à la cuisson, à la préparation d'aliments, ou à d'autres usages domestiques, quelle que soit leur origine et qu'elles soient fournies par un réseau de distribution, à partir d'un camion-citerne ou d'un bateau-citerne, en bouteilles ou en conteneurs ; b) toutes les eaux utilisées dans les entreprises alimentaires pour la fabrication, la transformation, la conservation ou la commercialisation de produits ou de substances destinés à la consommation humaine, à moins que les autorités nationales compétentes n'aient établi que la qualité des eaux ne peut affecter la salubrité de la denrée alimentaire finale ». Dans la terminologie internationale (Protocole de Londres, 1999, art. 2, 2a), la définition est plus courte et plus englobante, sans contradiction avec la précédente : "Toute eau utilisée ou destinée à être utilisée par l'homme pour la consommation, pour la cuisson et la préparation des aliments, pour l'hygiène corporelle ou à des fins similaires ». La législation française reprend le décret européen (2001) et remplace " eau potable " par " eaux destinées à la consommation humaine ", mais elle conserve le terme eau potable dans plusieurs domaines, par exemple celui des captages (d'eau potable). Quant à la législation fédérale allemande (2001), elle transpose la directive de 1998 et crée un "règlement de l'eau potable " (Trinkwasserverordnung) s'appliquant à « l'eau destinée à la consommation humaine ", qui comprend deux catégories distinctes : 1) l'eau potable (Trinkwasser) ;2) l'eau destinée à l'industrie alimentaire (Wasser für Lebensmittelbetriebe)! Autrement dit, l'allemand opère un découpage conceptuel différent et " eau potable » 
devient un hyponyme de " eau destinée à la consommation humaine". On comprend alors pourquoi il n'est pas simple d'établir des équivalences quadrilingues et pourquoi le principe des axies de la plate-forme LexALP peut être utile. Ce type de situation se retrouve d'ailleurs avec le slovène, langue slave, dont le découpage conceptuel est souvent différent de celui des langues romanes ou de l'allemand.

\section{Conclusion}

Dans la figure 2 ci-dessous se trouve la page d'accueil de la banque de termes LexALP déjà accessible au public ${ }^{(5)}$. L'interface de recherche se trouve à gauche et une recherche avancée permet de préciser sa requête.

\section{Figure 2}

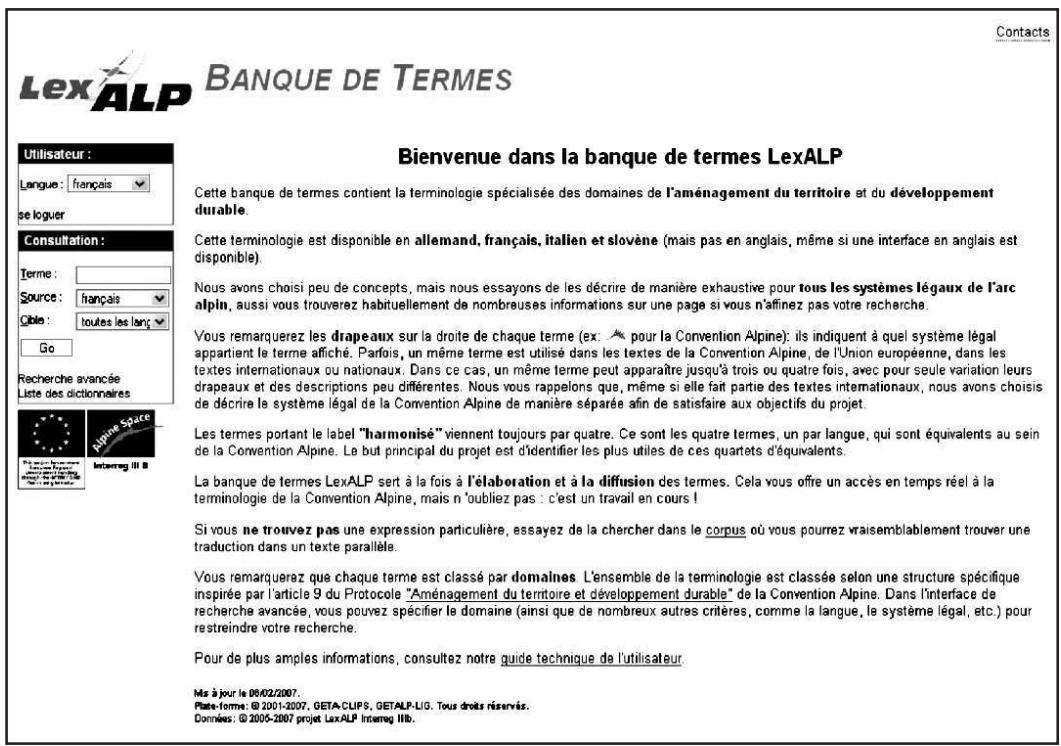

(5) http://www.eurac.edu/lexalp : cliquer sur termbank pour ouvrir l'interface de recherche dans la base de données terminologiques. Attention : les termes ne seront officiellement validés que début 2008. 
Pour l'instant la base est accessible mais elle est en phase d'édition et les fiches, dans leur grande majorité, n'ont pas encore été validées. De nombreuses corrections vont être apportées. L'harmonisation devrait être finie début 2008.

En facilitant la communication entre les acteurs politiques et juridiques des pays de l'arc alpin, un tel projet linguistique a des applications sociales et économiques évidentes, ce qui explique le soutien européen dont il a bénéficié. Nous espérons que les ressources produites pourront atteindre ses objectifs ambitieux. En tant qu'enseignants-chercheurs universitaires, c'est l'intérêt scientifique du projet qui nous a motivés : le travail terminographique effectué dans le cadre de ce projet a mené à une réflexion collective stimulante sur des questions de linguistique telles que le découpage conceptuel des différentes langues ou les rapports entre lexicologie et terminologie, sur des questions de méthodologie terminologique qui ont directement influencé les travaux, et aussi sur les deux domaines de spécialité complexes et passionnants que sont le droit avec ses composantes internationales et européennes et l'écologie associée au développement durable, qui porte notre avenir. Un autre intérêt pour nous était d'intégrer ce projet au programme des étudiants de dernière année, qui travailleront comme traducteurs dans quelques mois, et pour qui le travail sur projet réel ne peut être que très formateur. Enfin, c'est aussi notre représentation de l'Europe qui change : nos partenaires, leurs langues et leurs cultures, sont devenus plus familiers, tout comme les lieux des séminaires et les espaces alpins sur lesquels portent nos travaux. Les échanges que nous avons quotidiennement d'un bout à l'autre de l'arc alpin ont rendu l'espace européen à la fois plus proche et plus ouvert, et en tout cas bien réel. 
Élisabeth Lavault-Olléon est professeur à l'université de Grenoble (Université Stendhal). Elle y dirige depuis plus de 12 ans une formation de traducteurs, le Master (ex-DESS) "Traduction spécialisée multilingue ", ainsi quiun groupe de recherche, le GREMUTS (Groupe de recherche multilingue en traduction spécialisée) qui organise régulièrement des journées d'étude et des colloques sur la traduction. Elle est l'auteur de Fonctions de la traduction en didactique des langues, (Paris, Didier Érudition, 1985, 2 éd. 1998), de Traduction spécialisée : pratiques, théories, formations (Bern, Peter Lang, 2007) et de nombreux articles sur la traduction spécialisée et la didactique de la traduction.

Elisabeth.Lavault@u-grenoble3.fr

Claire Allignol est diplômée de l'IÜD, l'institut de traduction et d'interprétation de l'université de Heidelberg (Allemagne). Ses langues de travail sont l'allemand et le russe. Après un emploi dans le groupe industriel Mannesmann, elle travaille en indépendante en Allemagne comme traductrice et rédactrice technique. Titulaire d'un doctorat français en linguistique appliquée allemande et traductologie, elle a enseigné à l'université de Grenoble, puis à l'ETI à Genève. Maître de conférences à l'université de Grenoble depuis 2002, elle enseigne la traduction technique, l'interprétation de liaison et la terminologie et participe aux travaux du Groupe de recherche multilingue en traduction spécialisée (GREMUTS) de l'université de Grenoble.

Claire.Allignol@u-grenoble3.fr 